Proceedings of the 2007 Winter Simulation Conference

S. G. Henderson, B. Biller, M.-H. Hsieh, J. Shortle, J. D. Tew, and R. R. Barton, eds.

\title{
MONTE CARLO METHODS FOR VALUATION OF RATCHET EQUITY INDEXED ANNUITIES
}

\author{
Ming-hua Hsieh \\ Department of Management Information Systems \\ National Chengchi University \\ NO. 64, Sec. 2, ZhiNan Rd. \\ Taipei, 11605, TAIWAN
}

\begin{abstract}
Equity Indexed Annuities (EIAs) are popular insurance contracts. EIAs provide the insured with a guaranteed accumulation rate on their premium at maturity. In addition, the insured may receive extra benefit if the return of the linked index is high enough. There are a few variations of EIAs. We consider two types of EIAs: compound ratchet and simple ratchet. Under the geometric Brownian motion assumption for the equity index, plain compound ratchet options is known to have closed form solutions, but plain simple ratchet option is not. In this paper, we derive a closed form solution for plain simple ratchet option. For more exotic options, Monte Carlo methods are usually used for their valuation. To improve their efficiency, we propose two control variates based on the analytical solutions for the price of plain ratchet options. The effectiveness of the proposed control variates is examined via numerical examples of a typical contract.
\end{abstract}

\section{INTRODUCTION}

An Equity Indexed Annuity contract provides the policyholder with a guaranteed minimum annual return and offers participation in the equity market. The returns to be credited are based on the index-linked return, which is tied to the performance of an equity price index such as the Standard and Poor's 500, the participation rate and the guaranteed minimum return. The participation rate determines how much of the index increases will be used to compute the index-linked return. With the guaranteed minimum return, the downside risk of the equity market is limited. EIAs usually have a maturity ranging from one to ten years, with seven years being typical.

There exists a few variations of EIA contracts. The major contract types include point-to-point (PTP), ratchet (which comes in simple and compound versions), and high water mark; see Hardy (2003) for more information. The ways to calculate the index-linked return is usually called

\author{
Yu-fen Chiu \\ Department of Money and Banking \\ National Chengchi University \\ NO. 64, Sec. 2, ZhiNan Rd. \\ Taipei, 11605, TAIWAN
}

indexation method or indexing. For PTP EIAs, the indexing is based on the growth of the two time points, which is the simplest indexation method. High water mark EIAs have the feature similar to a lookback option. They choose the maximum index level over the entire term of an annuity for calculating payoff at maturity. Under the ratchet contract design, the indexing is usually evaluated annually based on the index growth during each time period. With a compound ratchet (CR), the returns in each time period are compounded, while the returns of each period of a simple ratchet (SR) are summed arithmetically. These types of contracts are more popular than the traditional life insurance products. Hence, their fair value and hedging strategies are crucial for life insurance companys. The valuation methods of EIAs has been discussed in a few papers: Tiong (2000) valued a contract slightly different to the usual contract design under the assumptions within the Black-Scholes framework. Lee (2003) used the method of Esscher transforms (similar to exponential twisted change-of-measures) to derive the explicit pricing formulas for four types of EIAs embedded with path-dependent options. The effects of stochastic interest rates and mortality were explored in Lin and Tan (2003).

In this paper, we focus on valuation methods for ratchet EIA contracts. In particular, we will discuss valuation methods for the following 4 types of ratchet EIA contracts. For most ratchet EIAs, the index participation is evaluated annually. To simplify the presentation, we assume this convention.

Let $T$ be the maturity of a ratchet EIA contract and $S(t)$ be the linked-index at time $t \leq T$. We set

$$
R_{t}=\frac{S(t)}{S(t-1)}, \quad t=1, \ldots, T
$$

which are the annual returns of linked-index. The effective annual returns of the EIA contract are defined as

$$
\tilde{R}_{t}=1+\min \left(\max \left(\alpha\left(R_{t}-1\right), f\right), c\right)
$$




\section{Hsieh and Chiu}

where $f$ is the guarantee rate, $c$ is the cap rate, and $\alpha$ is the participation rate in the linked-index.

With these notations, we are ready to define the payoffs of the first two types of ratchet EIA contract under consideration.

Definition 1 The payoff at maturity $T$ of a compound ratchet EIA contract is

$$
R_{c r}=\prod_{t=1}^{T} \tilde{R}_{t}
$$

when the initial investment is 1.

Definition 2 The payoff at maturity $T$ of a simple ratchet EIA contract is

$$
R_{s r}=1+\sum_{t=1}^{T}\left(\tilde{R}_{t}-1\right)=1-T+\sum_{t=1}^{T} \tilde{R}_{t}
$$

when the initial investment is 1.

Some ratchet EIA contracts offer not just annual guarantee rate $f$, but also a guarantee at maturity. This type of guarantee is sometimes called "life of contract" guarantee. Let the initial investment is $P$, of which $P(1-\beta)$ is deducted at beginning for covering expenses and insurance premium. A typical value for $\beta$ is $90 \%$. If the maturity guarantee promises an annual guarantee rate $g$, then the payoffs of the other two ratchet EIA contracts are defined as follows.

Definition 3 The payoff at maturity $T$ of a compound ratchet EIA contract with maturity guarantee is

$$
P \cdot \max \left(R_{c r}, \beta(1+g)^{T}\right)
$$

when the initial investment is $P$.

Definition 4 The payoff at maturity $T$ of a simple ratchet EIA contract with maturity guarantee is

$$
P \cdot \max \left(R_{s r}, \beta(1+g)^{T}\right)
$$

when the initial investment is $P$.

Hardy (2004) also discussed the valuation methods for the four types of ratchet EIA contracts defined above. The methods she considered included closed form formula, tree approach, and Monte Carlo simulation. She derived a closedform formula for plain compound ratchet EIAs, and argued that plain simple ratchet EIAs are not analytically tractable and proposed a pricing method utilizing a non-combining trinomial tree. For compound and simple ratchet EIAs with maturity or "life of contract" guarantee, Hardy used Monte Carlo methods as valuation tool. In this paper, we derive a closed form solution for plain simple ratchet option. Based on the closed form solutions for simple and compound EIA contracts, we are able to suggest two effective control variates for pricing ratchet EIAs with maturity guarantee.
The paper is organized as follows. In Section 2, we describe the risk neutral valuation formulas for the contracts under consideration and derive the pricing formulas for compound and simple ratchet EIA contracts. In Section 3, we use the payoffs of compound and simple ratchet EIAs as control variates for valuation of ratchet EIA contracts with maturity guarantee. Finally, Section 4 offers some concluding remarks and some directions for future works.

\section{VALUATION FORMULAS FOR COMPOUND AND SIMPLE RATCHET CONTRACTS}

Most of the previous research (Hardy 2004, Lee 2003, Tiong 2000, Gerber and Shiu 2003) adopted the Black and Scholes assumptions (Black and Scholes 1973) for the linked-index and interest rate. That is, the linked-index $S(t)$ follows the geometric Brownian motion and the interest rate $r$ is constant. In particular, under the risk-neutral measure or martingale measure, it assumes

$$
\begin{aligned}
d S(t) & =r S(t) d t+\sigma S(t) d z(t) \\
d B(t) & =r B(t) d t
\end{aligned}
$$

where $z(t)$ is a standard Brownian motion, $\sigma$ is the volatility of the linked index (which is constant), and $B(t)$ denotes the money market account.

Assume the market defined in (7) is complete, then based on the risk neutral valuation principle (see, for example, Harrison and Kreps 1979 and Harrison and Pliska 1981), the prices of the EIA contracts considered in Section 1 can be represented as expections. More specific, the price of a compound ratchet EIA contract is

$$
V_{c r}=E\left[e^{-r T} R_{c r}\right]
$$

and the price of a simple ratchet EIA contract is

$$
V_{s r}=E\left[e^{-r T} R_{s r}\right]
$$

For a compound ratchet EIA contract with maturity guarantee, its theoretical (no arbitrage) price is

$$
E\left[e^{-r T} P \cdot \max \left(R_{c r}, \beta(1+g)^{T}\right)\right],
$$

when the initial investment is $P$; and for a simple ratchet EIA contract with maturity guarantee, its theoretical price is

$$
E\left[e^{-r T} P \cdot \max \left(R_{s r}, \beta(1+g)^{T}\right)\right],
$$

when the initial investment is $P$.

Suppose the linked-index pays a continuous dividend yield at a constant rate $d$ per year. It is well known that, under the risk neutral measure (pricing measure), $\log \left(R_{t}\right)$ 


\section{Hsieh and Chiu}

are independent normal random variables with parameters $r-d-\sigma^{2} / 2$ and $\sigma^{2}$ (Hull 2006). Now using (2) we can get

$$
\tilde{R}_{t}=(1-\alpha)+\alpha \min \left(\max \left(f_{\alpha}, R_{t}\right), c_{\alpha}\right),
$$

where $f_{\alpha}=1+f / \alpha$ and $c_{\alpha}=1+c / \alpha$. Set

$$
X_{t}=\min \left(\max \left(f_{\alpha}, R_{t}\right), c_{\alpha}\right) .
$$

Then it is easy to see that $X_{t}$ 's are independent censored lognormal random variables with censored values $f_{\alpha}$ and $c_{\alpha}$.

We use (3) and (8) to obtain

$$
\begin{aligned}
V_{c r} & =E\left[e^{-r T} \prod_{t=1}^{T}\left(1-\alpha+\alpha X_{t}\right)\right] \\
& =e^{-r T}\left(1-\alpha+\alpha E X_{1}\right)^{T}
\end{aligned}
$$

and use (4) and (9) to obtain

$$
\begin{aligned}
V_{s r} & =E\left[e^{-r T}\left(1-T+\sum_{t=1}^{T} \tilde{R}_{t}\right)\right] \\
& =e^{-r T}\left[(1-\alpha T)+\alpha T E X_{1}\right]
\end{aligned}
$$

Therefore, we just need the explicit formula of $E X_{1}$ to derive the explicit formulas for $V_{c r}$ and $V_{s r}$.

To compute $E X_{1}$, we first write

$E X_{1}=f_{\alpha} P\left(R_{1} \leq f_{\alpha}\right)+E\left[R_{1} ; f_{\alpha} \leq R_{1} \leq c_{\alpha}\right]+c_{\alpha} P\left(R_{1} \geq c_{\alpha}\right)$.

Then, by representing $R_{1}$ as $e^{r-d-\sigma^{2} / 2+\sigma N(0,1)}$ and letting

$$
d_{1}=\frac{\log f_{\alpha}-r+d}{\sigma}+\frac{\sigma}{2},
$$

and

$$
d_{2}=\frac{\log c_{\alpha}-r+d}{\sigma}+\frac{\sigma}{2}
$$

we obtain

$$
\begin{aligned}
& P\left(R_{1} \leq f_{\alpha}\right)=P\left(N(0,1) \leq d_{1}\right)=\Phi\left(d_{1}\right) \\
& P\left(R_{1} \geq c_{\alpha}\right)=P\left(N(0,1) \geq d_{2}\right)=\Phi\left(-d_{2}\right)
\end{aligned}
$$

and

$$
\begin{aligned}
E\left[R_{1} ; f_{\alpha} \leq R_{1} \leq c_{\alpha}\right] & =\int_{d_{1}}^{d_{2}} e^{r-d-\sigma^{2} / 2+\sigma z} \phi(z) d z \\
& =e^{r-d}\left[\Phi\left(d_{2}-\sigma\right)-\Phi\left(d_{1}-\sigma\right)\right]
\end{aligned}
$$

where $\phi(\cdot)$ and $\Phi(\cdot)$ are the density function and the cumulative distribution function of standard normal random variable, respectively.

Combining these three terms, we get the explicit formula for $E X_{1}$ :

$$
f_{\alpha} \Phi\left(d_{1}\right)+c_{\alpha} \Phi\left(-d_{2}\right)+e^{r-d}\left[\Phi\left(d_{2}-\sigma\right)-\Phi\left(d_{1}-\sigma\right)\right]
$$

With (18), (14), and (15), the following two propositions are straightforward. Note that the pricing formula for the compound ratchet EIAs had been derived in the literature; see, for example, Hardy (2004).

Proposition 1 The explicit pricing formula for the compound ratchet EIA contracts is

$$
\begin{array}{r}
V_{c r}=e^{-r T}\left\{1-\alpha+\alpha\left(f_{\alpha} \Phi\left(d_{1}\right)+c_{\alpha} \Phi\left(-d_{2}\right)\right.\right. \\
\left.\left.+e^{r-d}\left[\Phi\left(d_{2}-\sigma\right)-\Phi\left(d_{1}-\sigma\right)\right]\right)\right\}^{T}
\end{array}
$$

Proposition 2 The explicit pricing formula for the simple ratchet EIA contracts is

$$
\begin{array}{r}
V_{s r}=e^{-r T}\left\{1-\alpha T+\alpha T\left(f_{\alpha} \Phi\left(d_{1}\right)+c_{\alpha} \Phi\left(-d_{2}\right)\right.\right. \\
\left.\left.+e^{r-d}\left[\Phi\left(d_{2}-\sigma\right)-\Phi\left(d_{1}-\sigma\right)\right]\right)\right\}
\end{array}
$$

We use the formulas in Proposition 1 and 2 to compute the theoretical prices of typical ratchet EIA contracts. Tables 1 and 2 summarize the results. From these results, it is easy to see that simple ratchet EIAs is cheaper than compound ratchet EIAs.

Table 1: Theoretical price of a typical compound ratchet EIA contract $(T=7, P=100, f=0, \sigma=25 \%, r=6 \%$, and $d=2 \%$.)

\begin{tabular}{|c|rrrrr|}
\hline$\alpha \backslash c$ & 0.10 & 0.15 & 0.20 & 0.30 & 0.40 \\
\hline 0.6 & 85.937 & 93.008 & 98.152 & 104.111 & 106.654 \\
0.8 & 87.601 & 96.600 & 104.043 & 114.568 & 120.591 \\
1.0 & 88.660 & 99.004 & 108.216 & 122.891 & 132.897 \\
1.2 & 89.391 & 100.714 & 111.290 & 129.512 & 143.465 \\
\hline
\end{tabular}

Table 2: Theoretical price of a typical simple ratchet EIA contract. ( $T=7, P=100, f=0, \sigma=25 \%, r=6 \%$, and $d=2 \%$.)

\begin{tabular}{|c|rrrrr|}
\hline$\alpha \backslash c$ & 0.10 & 0.15 & 0.20 & 0.30 & 0.40 \\
\hline 0.6 & 83.685 & 89.115 & 92.846 & 96.964 & 98.661 \\
0.8 & 84.996 & 91.738 & 96.918 & 103.727 & 107.384 \\
1.0 & 85.820 & 93.448 & 99.685 & 108.740 & 114.396 \\
1.2 & 86.383 & 94.642 & 101.666 & 112.525 & 119.987 \\
\hline
\end{tabular}




\section{Hsieh and Chiu}

\section{CONTROL VARIATES FOR RATCHET EIAS WITH MATURITY GUARANTEE}

In this section, our goal is to compute the prices of compound and simple rachet EIA contacts with "life of contract" guarantees. There are no known explicit pricing formulas for these insurance products. Thus, numerical methods are used to compute there values. We shall use Monte Carlo methods to do the task.

We begin with a description of the parameters of the ratchet EIA contracts under consideration. The contract maturity $T=7$, initial investment $P=100$, floor rate $f=0$, the volatility of the linked-index $\sigma=25 \%$, interest rate $r=6 \%$, dividend rate of the linked-index $d=2 \%$, net investment ratio $\beta=90 \%$ and the guarantee rate at maturity $g=3 \%$. These parameters are typical and were also used in Hardy (2004).

We simulate 1000 independent runs of $\left(\tilde{R}_{1}, \cdots, \tilde{R}_{T}\right)$. From these 1000 simulated paths, we can easily obtain 1000 independent replications of $e^{-r T} P \cdot \max \left(R_{c r}, \beta(1+g)^{T}\right)$ and $e^{-r T} P \cdot \max \left(R_{s r}, \beta(1+g)^{T}\right)$. Based on these independent copies, standard point estimates of $E\left[e^{-r T} P \cdot \max \left(R_{c r}, \beta(1+\right.\right.$ $\left.\left.g)^{T}\right)\right]$ and $E\left[e^{-r T} P \cdot \max \left(R_{s r}, \beta(1+g)^{T}\right)\right]$, and their standard errors are computed and presented in Table 3 and Table 4.

Table 3: Theoretical price of a compound ratchet EIA contract with "life of contract" guarantee computed by naive Monte carlo method. The upper table contains point estimates and the lower table contains their standard errors.

\begin{tabular}{|r|rrrrr|}
\hline$\alpha \backslash c$ & 0.10 & 0.15 & 0.20 & 0.30 & 0.40 \\
\hline 0.6 & 86.344 & 93.496 & 98.647 & 104.710 & 107.364 \\
0.8 & 87.989 & 97.107 & 104.634 & 115.161 & 121.326 \\
1.0 & 89.048 & 99.501 & 108.874 & 123.627 & 133.624 \\
1.2 & 89.788 & 101.231 & 111.941 & 130.437 & 144.388 \\
\hline \hline 0.6 & 0.299 & 0.453 & 0.580 & 0.764 & 0.864 \\
0.8 & 0.318 & 0.498 & 0.673 & 0.961 & 1.170 \\
1.0 & 0.330 & 0.526 & 0.733 & 1.116 & 1.429 \\
1.2 & 0.338 & 0.547 & 0.771 & 1.239 & 1.654 \\
\hline
\end{tabular}

Table 4: Theoretical price of a simple ratchet EIA contract with "life of contract" guarantee computed by naive Monte carlo method. The upper table contains point estimates and the lower table contains their standard errors.

\begin{tabular}{|r|rrrrr|}
\hline$\alpha \backslash c$ & 0.10 & 0.15 & 0.20 & 0.30 & 0.40 \\
\hline 0.6 & 84.567 & 90.194 & 94.148 & 98.612 & 100.452 \\
0.8 & 85.874 & 92.865 & 98.255 & 105.502 & 109.478 \\
1.0 & 86.661 & 94.627 & 101.091 & 110.582 & 116.647 \\
1.2 & 87.184 & 95.829 & 103.141 & 114.419 & 122.326 \\
\hline \hline 0.6 & 0.239 & 0.336 & 0.416 & 0.525 & 0.582 \\
0.8 & 0.250 & 0.359 & 0.454 & 0.605 & 0.705 \\
1.0 & 0.256 & 0.374 & 0.477 & 0.655 & 0.789 \\
1.2 & 0.259 & 0.382 & 0.494 & 0.688 & 0.847 \\
\hline
\end{tabular}

The accuracy of the point estimates in Table 3 and Table 4 are not very satisfactory. Of course, the accuracy of these point estimates can be improved by increasing the number of simulation runs. But, we prefer to apply the variance reduction techniques of control variate (see, e.g., Bratley, Fox, and Schrage 1983 and Law and Kelton 2000). In particular, we take advantage of known pricing formulas for the rachet EIA contacts without "life of contract" guarantees and select two control variates

$$
C_{1}=e^{-r T} \tilde{R}_{c r}-V_{c r}
$$

and

$$
C_{2}=e^{-r T} \tilde{R}_{s r}-V_{s r}
$$

Using the same 1000 replications of $\left(\tilde{R}_{1}, \cdots, \tilde{R}_{T}\right)$, we can also obtain 1000 independent replications of $C_{1}$ and $C_{2}$. Let $\lambda_{1}$ and $\lambda_{2}$ be any real numbers and set

$$
\begin{aligned}
Y\left(\lambda_{1}, \lambda_{2}\right) & =e^{-r T} P \cdot \max \left(R_{c r}, \beta(1+g)^{T}\right)-\lambda_{1} C_{1}-\lambda_{2} C_{2} \\
W\left(\lambda_{1}, \lambda_{2}\right) & =e^{-r T} P \cdot \max \left(R_{s r}, \beta(1+g)^{T}\right)-\lambda_{1} C_{1}-\lambda_{2} C_{2}
\end{aligned}
$$

Since $E C_{1}=0$ and $E C_{2}=0$, it is easy to see that $E\left[Y\left(\lambda_{1}, \lambda_{2}\right)\right]$ and $E\left[W\left(\lambda_{1}, \lambda_{2}\right)\right]$ equal to the theoretical prices of the compounded and simple ratchet EIAs with "life of contract" guarantee. Therefore, they provide alternative means of computing the prices. The optimal (variance-minimizing) weights of the control variates can be represented by the related covariance terms (Law and Kelton 2000) and are estimated by the sample covariances.

It turns out that these two control variates are quite effective. Table 5 and Table 6 show the results. These results indicate that the accuracy of the estimates has been improved significantly with the selected control variates.

Table 5: Theoretical price of a compound ratchet EIA contract with "life of contract" guarantee computed by Monte carlo method with control variates $C_{1}$ and $C_{2}$. The upper table contains point estimates and the lower table contains their standard errors.

\begin{tabular}{|r|rrrrr|}
\hline$\alpha \backslash c$ & 0.10 & 0.15 & 0.20 & 0.30 & 0.40 \\
\hline 0.6 & 86.121 & 93.182 & 98.320 & 104.273 & 106.812 \\
0.8 & 87.731 & 96.712 & 104.156 & 114.676 & 120.696 \\
1.0 & 88.757 & 99.080 & 108.290 & 122.964 & 132.967 \\
1.2 & 89.475 & 100.775 & 111.349 & 129.571 & 143.522 \\
\hline \hline 0.6 & 0.018 & 0.019 & 0.020 & 0.021 & 0.021 \\
0.8 & 0.016 & 0.017 & 0.017 & 0.018 & 0.018 \\
1.0 & 0.015 & 0.016 & 0.016 & 0.016 & 0.016 \\
1.2 & 0.014 & 0.015 & 0.015 & 0.015 & 0.015 \\
\hline
\end{tabular}




\section{Hsieh and Chiu}

Table 6: Theoretical price of a simple ratchet EIA contract with "life of contract" guarantee computed by Monte carlo method with control variates $C_{1}$ and $C_{2}$. The upper table contains point estimates and the lower table contains their standard errors.

\begin{tabular}{|r|rrrrr|}
\hline$\alpha \backslash c$ & 0.10 & 0.15 & 0.20 & 0.30 & 0.40 \\
\hline 0.6 & 83.906 & 89.322 & 93.053 & 97.173 & 98.869 \\
0.8 & 85.170 & 91.898 & 97.078 & 103.886 & 107.545 \\
1.0 & 85.965 & 93.579 & 99.817 & 108.871 & 114.527 \\
1.2 & 86.510 & 94.755 & 101.779 & 112.637 & 120.099 \\
\hline \hline 0.6 & 0.021 & 0.023 & 0.024 & 0.025 & 0.025 \\
0.8 & 0.019 & 0.021 & 0.021 & 0.023 & 0.023 \\
1.0 & 0.018 & 0.020 & 0.020 & 0.021 & 0.021 \\
1.2 & 0.018 & 0.019 & 0.019 & 0.020 & 0.020 \\
\hline
\end{tabular}

To further quantify the effectiveness of each control variate, we define variance reduction ratio as follows.

$$
\operatorname{VRR}=\frac{\operatorname{Var}(\text { naive estimator })}{\operatorname{Var}(\text { estimator with control variate }(\mathrm{s}))}
$$

Because most of the computational effort was used to generate the sample paths of $\left(\tilde{R}_{1}, \cdots, \tilde{R}_{T}\right)$, the additional work needed to compute $C_{1}$ and $C_{2}$ is minor. Therefore, it seems reasonable to use VRR as a proxy of computational gain. Table 7 shows the results for the compound ratchet EIA contract with 'life of contract" guarantee; and Table 8 shows the results for the simple ratchet EIA contract with 'life of contract" guarantee. $C_{1}$ is every effective for the compound ratchet EIAs and $C_{2}$ is very effective for the simple ratchet EIAs. The combination of $C_{1}$ and $C_{2}$ are most effective in reducing the estimator's variances.

Table 7: VRR when $C_{1}$ and $C_{2}$ were used as control variates for computing the price of a compound ratchet EIA contract with "life of contract" guarantee. The upper panel contains VRR when $C_{1}$ and $C_{2}$ were used simultaneously, the middle one contains VRR when $C_{1}$ was used only, and the lower one contains VRR when $C_{2}$ was used only.

\begin{tabular}{|r|rrrrr|}
\hline$\alpha \backslash c$ & 0.10 & 0.15 & 0.20 & 0.30 & 0.40 \\
\hline 0.6 & 174.5 & 344.5 & 532.0 & 864.6 & 1066.4 \\
0.8 & 218.4 & 471.0 & 806.4 & 1561.9 & 2239.4 \\
1.0 & 254.1 & 576.0 & 1051.6 & 2291.7 & 3703.5 \\
1.2 & 283.4 & 666.1 & 1259.3 & 3011.8 & 5299.2 \\
\hline \hline 0.6 & 113.6 & 246.8 & 405.1 & 701.4 & 891.7 \\
0.8 & 148.9 & 349.6 & 624.5 & 1290.1 & 1908.8 \\
1.0 & 175.9 & 434.1 & 821.9 & 1894.0 & 3169.8 \\
1.2 & 197.3 & 508.5 & 992.4 & 2491.5 & 4549.4 \\
\hline \hline 0.6 & 43.2 & 44.5 & 38.2 & 31.3 & 27.6 \\
0.8 & 49.1 & 47.0 & 37.9 & 25.6 & 21.1 \\
1.0 & 52.5 & 47.9 & 37.2 & 23.1 & 17.3 \\
1.2 & 54.9 & 48.1 & 36.4 & 22.1 & 15.0 \\
\hline
\end{tabular}

Table 8: VRR when $C_{1}$ and $C_{2}$ were used as control variates for computing the price of a simple ratchet EIA contract with "life of contract" guarantee. The upper panel contains VRR when $C_{1}$ and $C_{2}$ were used simultaneously, the middle one contains VRR when $C_{1}$ was used only, and the lower one contains VRR when $C_{2}$ was used only.

\begin{tabular}{|r|rrrrr|}
\hline$\alpha \backslash c$ & 0.10 & 0.15 & 0.20 & 0.30 & 0.40 \\
\hline 0.6 & 129.5 & 220.1 & 309.2 & 446.3 & 525.5 \\
0.8 & 164.6 & 299.0 & 446.3 & 722.4 & 935.5 \\
1.0 & 191.5 & 366.5 & 568.7 & 985.0 & 1364.9 \\
1.2 & 215.1 & 423.6 & 678.0 & 1227.6 & 1778.8 \\
\hline \hline 0.6 & 128.7 & 121.9 & 81.0 & 50.3 & 41.1 \\
0.8 & 154.2 & 116.9 & 70.5 & 37.1 & 27.6 \\
1.0 & 164.7 & 109.9 & 65.0 & 32.0 & 22.1 \\
1.2 & 170.1 & 105.6 & 61.3 & 29.8 & 19.3 \\
\hline \hline 0.6 & 82.5 & 158.5 & 239.1 & 372.2 & 452.7 \\
0.8 & 110.3 & 221.6 & 351.0 & 611.9 & 822.2 \\
1.0 & 134.3 & 280.3 & 454.9 & 843.3 & 1212.3 \\
1.2 & 155.5 & 331.1 & 549.6 & 1055.8 & 1587.9 \\
\hline
\end{tabular}

\section{CONCLUDING REMARKS AND FUTURE WORKS}

In this paper, we introduced four types of ratchet EIA contacts: compound ratchet, simple ratchet, compound ratchet with "life of contract" guarantee and simple ratchet "life of contract" guarantee. We derived the explicit pricing formulas for compound ratchet and simple ratchet contracts. To our best knowledge, the pricing formula for the simple ratchet contract is new in the literature. For the products with "life of contract" guarantee, we suggest using their counterparts without "life of contract" guarantee as control variates. The numerical results show that these controls are quite effective. This also suggests that Monte Carlo methods can be a very efficient computational tool for pricing complex insurance products.

Our study also provides computational tools for analyzing the trade-off among various parameters when the insurance companys design ratchet EIA contracts. For example, the following information can be obtained from our study:

1. The cost difference between compound or simple ratchet;

2. The effect of participation rate $\alpha$ on cost;

3. The effect of ceiling rate $c$ on cost;

4. The effect of floor rate $f$ on cost; and

5. The effect of the "life of contract" guarantee rate $g$ on cost. 


\section{Hsieh and Chiu}

Finally, we suggest a few directions for future research:

1. Simulate the linked index under a more complicated model, such as local volatility or regime switching Markov model and test the efficiency of the proposed control variates.

2. Expand the model to include the surrender (withdraw) model. The surrender behavior of the insured usually depends on interest rate level and the performance of the linked index.

3. Expand the model to include the mortality model.

4. Expand the model to include stochastic interest rate model.

\section{ACKNOWLEDGMENTS}

This research was supported by the National Science Council of Taiwan under contract number NSC95-2221-E-004-003.

\section{REFERENCES}

Black, F., and M. Scholes. 1973. The pricing of options and corporate liabilities. Journal of Political Economy 81:637-59.

Bratley, P., B. L. Fox, and L. Schrage. 1983. A guide to simulation. New York: Springer-Verlag.

Gerber, H., and E. Shiu. 2003. Pricing lookback options and dynamic guarantees. North American Actuarial Journal 7(1):48-67.

Hardy, M. 2004. Ratchet equity indexed annuities. In 14th Annual International AFIR Colloquium.

Hardy, M. R. 2003. Investment guarantees: Modelling and risk management for equity-linked life insurance. Wiley, New York.

Harrison, J. M., and D. M. Kreps. 1979. Martingales and arbitrage in multiperiod security markets. Journal of Economics Theory 20:381-408.

Harrison, J. M., and S. R. Pliska. 1981. Martingales and stochastic integrals in the theory of continuous trading. Stochastic Processes and their Applications 11:215-60.

Hull, J. C. 2006. Options, futures, and other derivatives securities, 6th edition. Prentice Hall International Editions.

Law, A. M., and W. D. Kelton. 2000. Simulation modeling \& analysis. 3rd ed. New York: McGraw-Hill, Inc.

Lee, H. 2003. Pricing equity-indexed annuities with pathdependent options. Insurance, Mathematics, and Economics 33(3):677-690.

Lin, S. X., and K. S. Tan. 2003. Valuation of equity indexed annuities under stochastic interest rates. North American Actuarial Journal 6:72-91.

Tiong, S. 2000. Valuing equity-indexed annuities. North American Actuarial Journal 4:149-170.

\section{AUTHOR BIOGRAPHIES}

MING-HUA HSIEH is an associate professor of the Department of Management Information Systems at National Chengchi University, Taiwan. From 1997 to 1999, he was a software designer at Hewlett Packard company, California. $\mathrm{He}$ is a member of INFORMS. His research interests include simulation methodology and financial engineering. His e-mail address is <mhsieh@mis.nccu.edu.tw>.

YU-FEN CHIU is a Ph.D. candidate in the Department of Banking at National Chengchi University, Taiwan. Her research interests include risk management and financial engineering. Her e-mail address is $\mathrm{g} 1352508 @ \mathrm{nccu}$. edu.tw>. 